\title{
Tangence
}

\section{La sémiotique corporelle de Marivaux}

\section{Paul Fortier}

Numéro 60, mai 1999

L'éloquence du corps sous l'Ancien Régime

URI : https://id.erudit.org/iderudit/008081ar

DOI : https://doi.org/10.7202/008081ar

Aller au sommaire du numéro

Éditeur(s)

Presses de l'Université du Québec

ISSN

0226-9554 (imprimé)

1710-0305 (numérique)

Découvrir la revue

Citer cet article

Fortier, P. (1999). La sémiotique corporelle de Marivaux. Tangence, (60), 64-83. https://doi.org/10.7202/008081ar d'utilisation que vous pouvez consulter en ligne.

https://apropos.erudit.org/fr/usagers/politique-dutilisation/ 


\section{La sémiotique corporelle de Marivaux \\ Paul Fortier, Collège de Rimouski}

Je n'ai qu'à regarder une femme entre deux yeux, je vous dirai ce qu'elle sent et ce qu'elle sentira, le tout à une virgule près. Tout ce qui passe dans son cœur s'écrit sur son visage, et j'ai tant étudié cette écriture-là, que je la lis tout aussi couramment que la mienne.

Marivaux

Au siècle de Louis XIV, la société courtoise est un vaste théâtre où hommes et femmes se jouent une mutuelle comédie. Comme l'écrit Pascal, "la vie humaine n'est qu'une illusion perpétuelle; on ne fait que s'entre-tromper et s'entre-flatter [...]. L'homme n'est donc que déguisement, que mensonge et hypocrisie, et en soi-même et à l'égard des autres" ${ }^{1}$. Ce jeu de fauxsemblants a progressivement débordé Versailles pour se répandre dans les salons dont l'attrait croît de jour en jour. Ainsi, lorsqu'en 1710 il arrive à Paris pour y prendre la plume, lorsque Fontenelle et La Motte l'introduisent dans le cercle de Madame de Lambert, le jeune Marivaux se rend-il rapidement compte que la folie du théâtre s'est emparée de tous. Philosophe et fin observateur des hommes, Marivaux a consacré une partie de ses romans de jeunesse, de ses essais et de ses journaux à réfléchir sur cette mascarade, à prendre position sur le theatrum mundi. Dénonçant le mensonge, il révèle un moyen pour y percer la vérité. Or cette éthique de la vérité lui échappe dans Le paysan parvenu. À un point tel, qu'il est incapable de mener son roman à terme.

\section{Le masque, usage et dénonciation}

Dans la foulée de la littérature baroque et de la commedia dell'arte, Pierre Carlet de Marivaux n'a pas manqué de voir dans

1 Blaise Pascal, Pensées, Paris, Garnier-Flammarion, 1976, p. 81 [éd. crit. L. Brunschwicg]. 
le thème du paraître un filon dont il a su exploiter la richesse. À chaque instant son personnage se cache derrière les artifices pour fausser la lecture de ses signes extérieurs; à tout moment il évite le regard des autres et s'enfuit derrière l'écran des fausses attitudes $^{2}$. Marivaux se sert de ce jeu de masques comme d'un ressort qu'il prend soin de bien tendre pour donner l'impulsion nécessaire aux aventures de ses romans de jeunesse et aux intrigues de plusieurs comédies. Pour mettre en scène cette continuelle dérobade, pour rendre ses personnages étrangers à euxmêmes, il utilise un ensemble varié de regards, de gestes, de mimiques et d'intonations d'emprunt; non seulement il met dans la bouche de ses personnages des paroles à double sens, mais il emploie aussi le déguisement au sens littéral du terme, c'està-dire en plaçant quelque maître dans des habits de valet, ou quelque valet dans des équipages de maître, en travestissant des femmes en hommes et des hommes en femmes.

Mais l'usage répété de ces artifices et tromperies ne fait pas de l'homme de lettres un apologiste de la mascarade. Loin d'être le défenseur des fourbes et des hypocrites, il est, au contraire, l'un de leurs plus durs accusateurs. En effet, ce ne sont pas des louanges qu'il adresse aux fripons mais bien un chapelet de reproches, et dans lesquels, dira Jacques d'Hondt, il "devient souvent sévère, cruel, impitoyable" 3 . En réalité, à l'image de la Parménie des Effets surprenants de la sympathie, la superficialité du monde civilisé le rebute:

L'éducation du monde ne donne à ceux qui la reçoivent presque toujours qu'une apparence vertueuse; on apprend ce qu'il faut faire pour paraître ou généreux, ou vertueux, et l'on apprend rarement ce qu'il faut faire pour le devenir véritablement: on s'attache à l'air et aux manières et quand on sait l'art de les rendre aimables, on se croit tel qu'il faut l'être. Le monde ne connaît que cette perfection superficielle, et toute sa sagesse n'est enfin que plus ou moins d'hypocrisie et d'autant plus dangereuse, que ceux qui la possèdent ne s'en aperçoivent pas. Ils se croient bons, parce qu'ils font par habitude une action qui a l'air de vertu. C'est une apparence qui les séduit,

2 Ce thème a d'ailleurs été largement étudié par Harold Schaad, dans son ouvrage Le thème de l'être et du paraître dans l'ouvre de Marivaux, Zurich, Juris-Verlag, 1969.

3 Jacques D'Hondt, "Hegel et Marivaux", Europe, n ${ }^{\circ} 451-452$, novembredécembre 1966, p. 332. 
leur délicatesse ne s'étend pas jusqu'à vouloir être bons par sentiment; leur cour ne connaît que le plaisir d'avoir trompé, il n'en sent point la honte; et l'estime qu'on a pour eux est le fruit d'une fourberie fine et secrète, ressemblante à la vertu, que l'éducation leur a inspirée, et dont ils sont, à la vérité, comme innocents, parce qu'ils s'abusent en abusant les autres. ${ }^{4}$

\section{La connaissance des hommes}

En accusant l'hypocrite, Marivaux ajoute qu'il existe un moyen de reconnaître le vrai visage des danseurs au bal masqué des échanges sociaux; il affirme qu'il est toujours possible d'éviter d'être entraîné dans leur tourbillon mystificateur. Certes, il sait "bien en gros que les hommes sont faux; que dans chaque homme il y en a deux, pour ainsi dire: l'un qui se montre, et l'autre qui se cache" 5 , mais il sait aussi qu'"il est dans tout le monde comme la possibilité plus ou moins forte ou faible de connaître les hommes à un certain point "6. Bien qu'il en parle dans son ouvre entière, c'est dans le Voyageur dans le Nouveau Monde qu'il développe de manière substantielle sa théorie sur la connaissance des hommes. Dans ce texte, plus que partout ailleurs, il lui "semble qu'on peut [...] vivre [avec les hommes] sans en être la dupe, et qu'il n'est pas si difficile de démêler ce qu'ils sont à travers ce qu'ils paraissent " ${ }^{7}$. Pour lui, rien ne peut empêcher que "ce qu'ils pensent dans le fond de l'âme perce toujours à travers ce qu'ils disent et ce qu'ils font " ${ }^{8}$.

Le narrateur de ce récit, un chevalier, est victime du mensonge d'autrui lorsqu'à sa grande surprise, il découvre que sa maîtresse le trompe avec son meilleur ami. Il provoque ce dernier en duel et, le croyant blessé à mort, il s'enfuit de par le monde. Dégoûté, honteux d'avoir été la dupe de gens à qui il avait accordé sa confiance, il s'en faut de peu qu'il ne devienne

4 Marivaux, Les effets surprenants de la sympathie, II, dans Euvres de jeunesse, Paris, Gallimard coll. "Bibliothèque de la Pléiade", 1972, p. 135 [éd, crit. F. Doloffre, avec le concours de C. Rigault].

5 Marivaux, "Le voyageur dans le Nouveau Monde", dans Le cabinet du philosophe, 6e f.-11 $\mathrm{e}$ f., dans Journaux et ouvres diverses, Paris, Garnier, coll. "Classiques", 1969, p. 390 [éd. crit. F. Deloffre et M. Gilot],

6 Marivaux, Réflexions sur l'esprit humain, dans JOD, p. 490.

7 Marivaux, "Le voyageur dans le Nouveau Monde", p. 396.

$8 \quad$ Ibid., p. 397. 
misanthrope. Mais il rencontre alors un étranger, un honnête homme, avec qui il se lie d'amitié. Ce nouvel ami l'invite à un voyage en mer, sorte de métaphore de l'exploration intérieure, au cours duquel le chevalier apprend à connaître l'homme. À son retour sur la terre ferme, il retrouve un Paris transformé, un Paris qu'il voit avec d'autres yeux, des yeux qui lui donnent accès au Monde vrai, un Monde vrai par lequel il entend des hommes vrais :

des hommes qui disent tout ce qu'ils pensent, et tout ce qu'ils sentent; qui ne valent pourtant pas mieux que nous, qui ne sont ni moins méchants, ni moins intéressés, ni moins fous que les hommes de notre monde; qui sont nés avec tous nos vices, et qui ne diffèrent d'avec nous que dans un seul point, mais qui les rend absolument d'autres hommes; c'est qu'en vivant ensemble, ils se montrent toujours leur âme à découvert, au lieu que la nôtre est toujours masquée. ${ }^{9}$

En faisant entrer son personnage dans ce nouveau monde, Marivaux procède à une véritable levée des masques:

en vous peignant ces hommes que j'ai trouvés, je vais vous donner le portrait des hommes faux avec qui vous vivez, je vais vous lever le masque qu'ils portent. Vous savez ce qu'ils paraissent, et non ce qu'ils sont. Vous ne connaissez point leur âme, vous allez la voir au visage, et ce visage vaut bien la peine d'être vu; ne fût-ce que pour n'être point la dupe de celui qu'on lui substitue, et que vous prenez pour le véritable. ${ }^{10}$

L'authenticité d'autrui, cette vérité qui apparaît aux yeux du chevalier, ce sont les différents instruments du langage non verbal qui la révèlent dans sa plus naïve ingénuité:

par ce Monde vrai, je n'entends pas des hommes qui prononcent précisément ce que je leur fais dire, leur naïveté n'est pas dans leurs mots [...]: elle est dans la tournure de leurs discours, dans l'air qu'ils ont en parlant, dans leur ton, dans leur geste, même dans leur regard: c'est dans tout ce que je dis là que leurs pensées se trouvent bien nettement, bien ingénument exprimées; des paroles prononcées ne seraient pas plus claires. Tout cela forme une langue à part qu'il faut entendre, que j'entendais alors dans les autres pour la première fois de ma vie, que j'avais moi-même parlée quelquefois, sans y

9 Ibid., p. 389.

10 Ibid., p. 389-390. 
prendre garde, et sans avoir eu besoin de l'apprendre, parce qu'elle est naturelle et comme forcée dans toutes les âmes. Langue qui n'admet point d'équivoque; l'âme qui la parle ne prend jamais un mot l'un pour l'autre. ${ }^{11}$

Si la percée à travers le masque se réalise par le comportement non verbal, il ne s'agit pas là de ces actions au cours desquelles l'âme impose son empire, mais bien d'échappées furtives de l'inconscient qui, à la surface du corps, tracent les signes de l'émotion. Parce que quelles que soient ses tentatives, l'homme ne peut à tout instant maintenir son corps sous la bride de sa volonté. Malgré la vigilance de la raison, la vérité sait donc faire du corps son porte-parole; elle sait "articuler" un discours muet, énoncer une "langue à part".

Malgré le couvert des bonnes manières, des jeux de rôles et des artifices de la vanité qui sur le corps s'appliquent comme un masque, il est donc toujours possible de saisir le moment furtif, l'élan spontané, véritable de l'expression naturelle, de l'indiscrétion du coeur. C'est alors que devant le voyageur s'ouvrent les portes du Monde vrai. Au cours de son intrusion dans le labyrinthe de la société civile, il voit les signes non verbaux constituer un fil d'Ariane qui l'empêche de se perdre. Savoir reconnaître la vérité prend alors la forme d'une activité tout autant ludique qu'utile.

Le spectacle donné à tout moment par autrui offre en effet un "plaisir singulier" à celui qui a découvert la clé de la vérité. "Je n'ai jamais été si content; je ne me suis jamais diverti de si bon coeur depuis ma découverte. Je suis à la comédie depuis le matin jusqu'au soir", affirme d'ailleurs le voyageur ${ }^{12}$.

Encore plus que la jouissance qu'elle procure, la lecture de l'autre s'inscrit comme une donnée essentielle de la réussite sociale. Marivaux la considère si importante qu'il en fait une question de vie et de mort:

Il faut [...] nécessairement qu[e le citoyen du monde] connaisse les hommes, il ne saurait se soutenir parmi eux qu'à cette condition-là.

Il va de tout pour lui d'être à certain point au fait de ce qu'ils ont pour savoir y accommoder ce qu'il est, pour juger d'eux, 
sinon finement, du moins au degré suffisant de justesse qui convient à son état et à la sorte de liaison ordinaire ou fortuite qu'il a avec eux.

Il y va toujours de sa fortune, toujours de son repos, souvent de son honneur, quelquefois de sa vie; quelquefois du repos, de l'honneur, de la fortune et de la vie des autres. ${ }^{13}$

Mais quoique soit présente "dans tout le monde" la possibilité d'accéder à cette connaissance, d'en savourer le plaisir et de profiter des avantages qu'elle procure, le fait demeure que l'ouverture des portes du Monde vrai est loin de représenter une tâche facile. Même si le corps livre indiscrètement la vérité, rien n'empêche que ses signes sont constamment court-circuités par ceux du mensonge. Or, comment faire la part du vrai et la part du faux, comment discerner l'action de la passion de l'âme, au sens que Descartes confère à ces termes? À quel niveau s'opère le décodage de cette si complexe sémiotique incarnée? C'est sur ces questions que portera maintenant notre discussion.

\section{Le sentiment, code de lecture et de comportement}

Durant la période pendant laquelle écrit Marivaux, le discours social fait de la communication non verbale le lieu d'interférences de deux forces opposées. D'un côté, la raison tente d'imposer sa volonté en ordonnant au corps de se contenir et de simuler; de l'autre l'émotion essaie dans un élan impulsif d'émerger à la surface charnelle. D'une part se mettent en place des signes conventionnels; de l'autre, des signes naturels. À leur façon, les deux systèmes sémiotiques traduisent le dilemme classique de l'esprit et du corps, de l'action et de la passion.

Comme c'est le cas des signes linguistiques, les signes conventionnels du comportement non verbal, ceux produits par l'action de l'âme, reposent essentiellement sur un ensemble de règles que partagent les membres d'une même culture. Ces règles, nous les retrouvons inscrites dans un foisonnement d'ouvrages divers consacrés aux traités de civilité et de savoir-vivre, aux grammaires des expressions, comme celle de Charles Le Brun ${ }^{14}$, ou encore

13 Marivaux, Réflexions sur l'esprit humain, op. cit., p. 477.

14 Charles Le Brun, "Conférence sur l'expression générale et particulière [tenue en 1668 à l'Académie royale de peinture et de sculpture]", Nouvelle revue de psychanalyse, $\mathrm{n}^{\circ} 21$, printemps 1980, p. 95-121. 
dans l'ensemble des codes de l'actio formulés dans les traités de rhétorique. Arbitraires, ces signes prennent valeur de symboles et sont régis par un système d'intelligibilité où prédominent la loi, la logique et la raison. À l'opposé, les signes naturels de la communication non verbale, ceux émis par les passions de l'âme, ne sont tributaires d'aucune règle ni convention. Ils sont échangés dans l'immédiateté, sous l'impulsion de l'émotion et du sentiment.

L'interprète voulant faire sienne la signification de l'un ou l'autre de ces systèmes sémiotiques doit mettre son acte de décodage au diapason de l'encodage. Ainsi, celui qui veut décrypter les signes symboliques doit-il faire appel à son raisonnement logique, à son intelligence cognitive ou ses habitudes culturelles : son rapport au signe est alors médiatisé par la convention. De l'autre côté, celui qui veut appréhender les signes dits "naturels" doit laisser la lecture aux bons soins de son instinct et de son intelligence intuitive: le rapport au signe est alors immédiat, instantané. C'est l'inconscient qui reçoit le message d'un autre inconscient, le sentiment qui capte le sentiment d'autrui.

C'est cette forme de communication de sentiment à sentiment que déploie Marivaux lorsqu'il met en scène l'un ou l'autre de ses Ulysses du Monde Vrai. En effet, ce n'est que lorsque son spectateur place sa lecture sur le registre du sentiment qu'il est à même de reconnaître la vérité des signes naturels cachée sous le mensonge des signes artificiels. Le sentiment, et lui seul, "fait juger sainement des faux ou des vrais mouvements qu'on donne au coeur" ${ }^{15}$. Notons ici que Marivaux utilise souvent le terme "mouvement" au sens que lui attribuait Descartes. À sa suite il admet que les mouvements de l'âme agitent les esprits animaux qui impriment au corps l'expression physique de l'émotion.

Le sentiment, selon la sémantique marivaudienne, est une sorte d'intuition, d'instinct, une vision immédiate de la vérité. Certes, une telle vision peut apparaître trouble, vague, "embarrassée", au regard de l'intelligence cognitive, mais cela ne l'empêche pas d'être infaillible. Autrement dit, l'instinct ne sait ni pourquoi ni comment, mais il sait toujours quoi et avec précision.

Cet instinct est donc [...] une sorte de sentiment qui porte instruction sans clarté; c'est une vue trouble de l'âme embarrassée

15 Marivaux, Les effets surprenants de la sympathie, op. cit., p. 3. 
dans ses organes; en un mot, l'instinct est à l'âme humaine un sentiment non déployé, qui lui prouve la vérité des choses qu'elle aperçoit nettement, en lui montrant un mystère obscur des dépendances qu'elles ont avec d'autres. ${ }^{16}$

En s'ouvrant de la sorte à l'instinct, à l'intuition, au cœur, au mouvement ou au sentiment (les termes sont interchangeables), Marivaux invalide à plus d'une occasion la raison comme voie d'accès à la connaissance. Nul besoin des méthodes, des techniques et des arts comme dans le système élaboré par Cureau de la Chambre ${ }^{17}$ au $\mathrm{xvII}^{\mathrm{e}}$ siècle, pour réaliser le décryptage d'autrui. Ainsi l'homme apprend-il le cour de l'homme "comme à son insu, sans qu'il y pense, sans que son expresse réflexion s'en mêle "18.

Seul instrument permettant le décodage de la vérité corporelle, le sentiment marivaudien se retrouve en chacun. C'est celui de l'enfance, celui de l'innocence, comme le suggère Marivaux dans un extrait capital, mais peu commenté, où il explique comment un enfant peut comprendre un discours sans connaître le code linguistique:

Un cerveau tendre, une âme neuve, vide d'idées, plus étonnée qu'elle ne le sera jamais des sons que nous articulons et qui la frappent, par conséquent plus attentive qu'on ne peut le dire à l'air et à la manière dont nous prononçons les mots, cherchant à savoir à quoi ils aboutissent et ce qu'ils signifient, et le cherchant avec une curiosité dont l'exactitude, la finesse et l'activité ne se retrouvent plus, et ne sont jamais attachées qu'aux premiers étonnements que l'âme éprouve; voilà vraisemblablement ce qui met encore un enfant en état de s'éclairer sur les mots de sa langue, voilà ce qui lui en révèle la signification, dont la connaissance, à mesure qu'il l'acquiert, l'introduit tout de suite dans l'étude imperceptible [...] de la connaissance des hommes. ${ }^{19}$

La connaissance de la vérité, ce n'est donc pas dans les mots que l'enfant l'appréhende, c'est dans l'air, dans la manière dont ils sont prononcés, c'est dans le champ de l'énonciation non verbale qui, dans cet extrait, paraît relever de l'instrument vocal ${ }^{20}$.

16 Marivaux, Pensée sur différents sujets, dans JOD, p. 71-72.

17 Marin Cureau de la Chambre, Les charactères des passions, 5 vol., p. 16401662.

18 Marivaux, Réflexions sur l'esprit bumain, op. cit., p. 478.

19 Ibid., p. 479.

20 Ce langage perçu par l'enfant de Marivaux ressemble à s'y méprendre à la langue primitive décrite par Rousseau et qui repose sur une prédominance 
Lire le sentiment, spontané, intuitif, libre de règles, de conventions et de préjugés, exige donc de retrouver la pureté de son cour d'enfant. Écouter son cour conduit à recouvrer la clarté originelle d'une perception que l'appareil symbolique du verbe n'a pas encore faussée. S'y ouvrir permet de sentir le naturel d'un geste, d'un regard, d'une mimique ou d'un soupir que les comédiens de la vie sociale essaient de fausser.

Plus encore qu'un moyen de lire la vérité, le sentiment devient ensuite la pierre d'assise de l'éthique comportementale marivaudienne. De même que le sentiment donne accès à la lecture du corps, de même doit-il être adopté en tant que code de son propre langage corporel. Or, se comporter au gré de son sentiment, c'est retrouver sa naïveté primaire; c'est regagner une franchise et une spontanéité que l'éducation avait mises au ban. Se laisser conduire par son instinct, c'est agir dans la totale liberté d'une enfance retrouvée, c'est répondre au rythme des mouvements véritables de son coeur, c'est se livrer de façon sincère et authentique.

\section{L’expérience du Paysan parvenu ${ }^{21}$}

Réduite à sa plus brève expression, le système marivaudien comprend deux vecteurs aux orientations distinctes. Ce qu'il faut voir est la vérité d'autrui, ce qu'il faut laisser voir est sa propre vérité. Cette hypothèse faisant du sentiment la voie royale de la vérité, Marivaux la mettra à l'épreuve dans Le paysan parvenu. En effet, beaucoup plus sérieuses que les pérégrinations d'un héros comique, ce que laisse pourtant supposer la tradition critique, les aventures de Jacob ne sont "point [...] une histoire forgée à plaisir" (I, p. 6). Elles forment plutôt l'objet d'un roman expérimental ${ }^{22}$.

vocalique: "Les passions [...] ont aussi leurs accens, et ces accens qui nous font tressaillir, ces accens auxquels on ne peut dérober son organe pénètrent par lui jusqu'au fond du cour, y portent malgré nous les mouvemens qui les arrachent, et nous font sentir ce que nous entendons" (Jean-Jacques Rousseau, Essai sur l'origine des langues, Paris, Gallimard, coll. "Folio Essais", 1990, p. 63 [éd. crit. J. Starobinski]).

21 Toutes les références au Paysan parvenu renvoient à l'édition de F. Deloffre et F. Rubellin, Paris, Bordas, "Classiques Garnier", 1992.

22 C'est l'expression que Jean Fabre utilise pour désigner la Vie de Marianne et le Paysan parvenu, et qui sera reprise dans le titre de l'article de Michèle Hirsh, intitulé "Le roman expérimental de Marivaux", dans Revue des sciences bumaines, vol. XXXIX, no 153, janvier-mars 1974, p. 103-124. 
Dans Paris, où se joue la plus grande comédie du monde, Marivaux choisit de placer un paysan venu de Champagne. Enfant naïf et rustique, c'est Jacob, cousin du héros picaresque ou descendant d'Arlequin, dont la mine est aussi attachante que sympathique. Le romancier lui donne comme seuls attributs ses dispositions naturelles, qualités avec lesquelles il voit et qu'en retour il fait voir, puis il l'observe vivre au sein d'un cercle d'hypocrites. Il juge si son sentiment lui fera éviter les pièges et si sa spontanéité saura le faire aimer; il examine s'il gardera ses vertus d'origine ou s'il se laissera corrompre par les conventions.

En prenant place dans un univers qui lui est totalement étranger, dans une société fondée sur le jeu des artifices et des fauxsemblants et dont il ne connaît aucunement les usages et conventions, il devient en quelque sorte un second "Voyageur dans le Nouveau Monde" 23. À l'image du héros du Monde vrai, Jacob, narrateur du récit-mémoires, dit n'être ni troublé ni confus devant l'espace culturel nouveau qui devant lui se déploie. Certes il le surprend à première vue, mais en bout de ligne ce "grand monde" le divertit bien plus qu'il ne l'étonne. Malgré la marginalité de sa condition, il se meut sur l'échiquier parisien sans contrainte ni difficulté; en dépit de son ignorance des règles du comportement civil, jamais son regard n'est trompé.

La raison de cette infaillibilité est qu'il fait du sentiment naturel son seul et unique juge. Nul n'est besoin selon lui de connaître les us et coutumes d'une société "pour être tout d'un coup au fait de certaines choses" (IV, p. 171): "il ne faut ni délicatesse ni usage du monde [...], il ne faut que des sens et j'en avais" (ibid.). Ainsi se laisse-t-il pénétrer de tout ce qu'il voit (I, 39), confie-t-il à son coeur la tâche de reconnaître l'indiscrétion du coeur d'autrui, ce qui est bien la façon la plus sûre et la plus rapide de lire la vérité des

23 Le lien entre ce récit inséré dans le Cabinet du philosophe et le roman du Paysan parvenu n'est pas fortuit. Selon la chronologie établie par Frédéric Deloffre, la publication hebdomadaire des feuilles dans lesquelles sont contenues le premier texte a commencé à la fin de janvier 1734, soit immédiatement après la parution de la seconde partie de la Vie de Marianne, et s'est terminée à la fin avril de la même année, moment où paraît la première partie du Paysan parvenu. Cet étroit rapport chronologique permet à Monsieur Deloffre de voir le Paysan parvenu comme une continuation du "Voyageur dans le Nouveau Monde"; hypothèse que nous partageons. 
74

sentiments ${ }^{24}$. En ses mots "il n'y a plus dans le cour de discrétion qui tienne, il dit en un quart d'heure ce que, suivant les règles de bienséance, il n'aurait osé dire qu'en un mois" (II, p. 93).

À l'instar du "Voyageur dans le Nouveau Monde", Jacob possède donc "ce talent de lire dans l'esprit des gens et de débrouiller leurs sentiments secrets" (II, 86). Sa fine sensibilité lui permet d'entendre la "langue à part" de la communication non verbale. Bien humblement, il précise: c'est "un don ${ }^{25}$ que j'ai toujours eu et qui m'a quelquefois servi" (ibid.). Clin d'œil ironique typique du personnage et de son auteur, cette proposition est loin de refléter la réalité. En fait, Jacob use de son talent à chacune des occasions qui se présentent à lui. Avec son cœur d'enfant, il sait à travers un sourire, un air, un ton, un silence, un geste ou une mine, faire la part du faux et du vrai. Il sent quand les autres s'affirment franchement ou quand ils lui jouent la comédie. Par cette traversée des apparences, il peut identifier ceux qui seront ses alliés ou ses ennemis.

Si la naïveté du regard de Jacob lui permet avec une sage lucidité de saisir la vérité d'autrui, son comportement, ignorant des conventions, pourrait en revanche l'exposer au ridicule, faire de lui un objet de sarcasme et de moquerie lorsqu'il s'offre au regard des autres.

Or ce n'est pas toujours le cas. Sa "présentation de soi", pour reprendre le titre d'un ouvrage d'Erwing Goffman, détient un énorme pouvoir de séduction auprès des femmes. Son discours non verbal, voie/voix de la franchise et de la naïveté, les prédispose immanquablement en sa faveur. Ainsi, l'une prendelle la liberté de porter sur lui un regard qu'elle devient sur le coup subjuguée par ses attraits naturels. Fait à noter, cette relation authentique entre la figure de l'enfant et la figure maternelle se déroule presque toujours dans un espace intime.

24 Ainsi se rapproche-t-il de Jean-Jacques Rousseau, qui dans les propos liminaires de ses Confessions écrira: "Je sens mon cour et je connais les hommes".

25 Henri Coulet (dans son édition du Paysan parvenu, Paris, Gallimard, coll. "Folio", 1981, p. 557) note de manière tout à fait intéressante que l'édition publiée par Duviquet avait arbitrairement substitué le mot "art" au terme "don". Ce qui est une erreur capitale puisque à la lumière des remarques que nous avons précédemment formulées, la distinction entre la technique et l'intuition était fondamentale dans la pensée de Marivaux. 
Le comportement de Jacob change radicalement lorsque dans un espace social il est mis au contact des hommes. Il semble à ce moment qu'il ne peut se dérober à l'ordre de la loi, de la règle et de la convention ${ }^{26}$. Alors, il s'oblige à se contenir, à tenir son idéal état d'enfant/paysan sous scellé. S'amorce ainsi, parallèlement à sa vie intime, une lente mais progressive métamorphose au cours de laquelle il procède à une symbolisation de sa communication verbale et non verbale. Se changer en traître, voilà le prix qu'il doit payer pour devenir un homme.

La métamorphose de Jacob commence dès les premiers jours de son expérience parisienne, c'est-à-dire lorsqu'il est reçu dans la maison de son seigneur. D'entrée de jeu son nouvel environnement oblitère ses origines paysannes en lui retirant le hâle que l'air de la campagne avait placé sur son visage. Puis petit à petit, Jacob commet des galanteries qui lui font nier ses origines (I, p. 12), et il se laisse aller à quelques mensonges non verbaux. À ces changements corporels se superpose une transformation d'ordre vestimentaire, comme celle que lui impose son hôtesse en lui fournissant un nouvel équipage; l'habit lui va si bien qu'il en perd son air villageois (I, p. 14). Survient ensuite une transformation sur le plan intellectuel: avec l'argent que lui donne Geneviève, Jacob prend des leçons d'écriture et d'arithmétique, signes d'une volonté de familiarisation avec les systèmes de symboles et de conventions (I, p. 23).

Le processus de transformation sera irréversible. Si Mademoiselle Habert aime la naïveté et la simplicité de Jacob, elle préfère cependant qu'il les cache lorsqu'il se présente au monde. Aussi commence-t-elle par lui demander de modeler son langage non verbal sur le $\operatorname{sien}^{27}$. Jacob ne se fait pas prier pour répondre aux ordres de sa future. En fait, il paraît se complaire

26 Sur le plan psychanalytique, le sujet s'y voit imposer un interdit entre le désir incestueux et son objet. Cet interdit survient au cours du "processus de symbolisation inaugural désigné par Lacan métaphore du Nom-du-Père" (Dor, Structure et perversion, Paris, Denoël, coll. "L'espace analytique", 1987, p. 148). À partir de ce moment, le sujet se voit bombardé de systèmes de signes et de signifiants, dont celui du langage, avec lesquels il doit composer.

27 "[T]u as dû remarquer que je n'ai rien dit chez notre hôtesse [Madame d'Alain] qui pût te faire connaître pour un domestique; elle n'aura pas non plus deviné que tu en es un; ainsi je te recommande, quand nous irons chez elle, de régler tes manières sur les miennes" (II, p. 79). 
76

dans cette mascarade: "Vertubleu! que je suis aise de toute cette manigance-là ", s'écrie-t-il (II, p. 80). Et effectivement il ne tarde pas à mettre ses conseils en pratique.

Si Jacob se plaît à copier et à emprunter des manières qui ne lui sont pas naturelles, il semble à ses yeux qu'il faille "encore ajuster une autre affaire" ( $\mathrm{ibid}$.). Et cette affaire gravite autour de la question de son nom. Avec la complicité de la cadette, il s'autorise à s'octroyer le Nom-du-Père. Audacieuse, subversive au plus haut point, cette décision, condition à ses yeux de la réussite sociale, marque le début de sa déroute personnelle:

qui voulez-vous que je sois? [demande-t-il à sa future] Voilà que vous me faites un monsieur, mais ce monsieur, qui sera-ce? Monsieur Jacob? Cela va-t-il bien? Jacob est mon nom de baptême, il est beau et bon ce nom-là; il n'y a qu'à le laisser comme il est, sans le changer contre un autre qui ne vaudrait pas mieux; ainsi je m'y tiens; mais j'en ai besoin d'autre; on appelle notre père le bonhomme la Vallée, et je serai monsieur de la Vallée son fils, si cela vous convient (ibid.).

$\mathrm{Au}$ moment où lui et sa future prennent pignon sur rue chez la d'Alain, du nom de Jacob il ne sera plus question: la cadette n'appellera Jacob "mon enfant" qu'à de rares occasions, préférant le nommer La Vallée ou Monsieur la Vallée. Le narrateur des mémoires agira de même: "Nous voilà à l'autre maison; et c'est d'ici qu'on va voir mes aventures devenir plus nobles et plus importantes; c'est ici où ma fortune commence: serviteur du nom de Jacob, il ne sera plus question que de monsieur de la Vallée" (II, p. 84). Enfant il n'est plus, homme il est devenu. C'est du moins ce qu'il pense.

L'entrée de Jacob dans l'ordre des symboles et des signifiants se développe au point où il transforme son comportement langagier. Il y a deux ou trois mois qu'il est à Paris mais il a déjà corrigé son accent villageois: "Jusqu'ici donc mes discours avaient toujours eu une petite tournure champêtre; mais il y avait plus d'un mois que je m'en corrigeais assez bien [...]; il est certain que je parlais meilleur français [...]. J'avais déjà acquis assez d'usage pour cela, et je crus devoir m'appliquer à parler mieux qu'à l'ordinaire" (II, p. 85). S'il a perdu son accent naturel, Jacob prend cependant soin de l'imiter lorsqu'il échange avec la cadette, sachant fort bien que celle-ci en goûte toujours la saveur: "je n'avais conservé cette tournure avec $\mathrm{M}^{\text {lle }}$ Habert, qu'à cause que je m'étais aperçu qu'elle me réussissait auprès d'elle, et 
que je lui avais dit tout ce qui m'avait plu à la faveur de ce langage rustique"( $i b i d$.).

Jacob pousse son jeu de faussaire jusqu'à l'injure en mentant auprès de celle sans qui il ne serait rien. Lors d'une scène entre lui, Agathe, Madame d'Alain et la cadette, il use de manigances et d'artifices pour plaire à chacune sans pour cela déplaire à aucune. Trait d'une manipulation machiavélique, son comportement non verbal jadis naturel n'est plus que poudre aux yeux et feu d'artifice.

Je redoublai d'agréments le plus qu'il me fut possible, pour entretenir Mademoiselle Habert dans les alarmes qu'elle prenait; mais comme il fallait qu'elle eût peur du goût qu'on avait pour moi, et non pas de celui qu'elle m'aurait senti pour quelqu'une de ces deux personnes, je me ménageai de façon que je ne devais lui paraître coupable de rien; elle pouvait juger que je n'avais point d'autre intention que de me divertir et non pas de plaire, et que, si j'étais aimable, je n'en voulais profiter que dans son cour, et non dans celui d'aucune de ces femmes.

Pour preuve de cela j'avais soin de la regarder très souvent avec des yeux qui demandaient son approbation pour tout ce que je disais; de sorte que j'eus l'art de la rendre contente de moi, de lui laisser ses inquiétudes qui pouvaient m'être utiles, et de continuer de plaire à nos deux hôtesses, à qui je trouvai aussi le secret de persuader qu'elles me plaisaient, afin de les exciter à me plaire à leur tour, et de les maintenir dans ce penchant qu'elles marquaient pour moi [...] (II, p. 86-87).

Le vocabulaire utilisé ici illustre bien à quel point Jacob se "ménage", veut "paraître", se comporte avec "soin", travaille avec "art", trouve le "secret de persuader". Parce qu'elle procède d'un "redoublement d'agréments", sa communication, non verbale autant que verbale, se trouve à des lieues d'une production naturelle des passions de l'âme. Bien au contraire, elle est devenue le résultat d'une action, d'une intention volontaire; elle n'est plus qu'ornement de la vérité, qu'une rhétorique de l'expression utilisée afin de convaincre et persuader.

Devenu l'incarnation du mensonge, Jacob substitue la feinte à la sincérité de son amour pour la cadette. À la question de celleci qui lui demande si elle peut croire en son amour, il lui répond affirmativement, mais d'un oui sans franchise et sans spontanéité. Sa rhétorique réussit à un point tel que Jacob devient la dupe de 
78

ses propres tromperies et que son sentiment tombe lui aussi sous le joug de la corruption:

Je me ressouviens bien qu'en lui parlant ainsi, je ne sentais rien en moi qui démentît mon discours. J'avoue pourtant que je tâchai d'avoir l'air et le ton touchant, le ton d'un homme qui pleure, et que je voulus orner un peu la vérité, et ce qui est singulier, c'est que mon intention me gagna tout le premier. Je fis si bien que j'en fus la dupe moi-même et je n'eus plus qu'à me laisser aller sans m'embarrasser de rien ajouter à ce que je sentais; c'était alors l'affaire du sentiment qui m'avait pris, et qui en sait plus que tout l'art du monde.

Aussi ne manquai-je pas mon coup; je convainquis, je persuadai si bien Mlle Habert, qu'elle me crut jusqu'à en pleurer d'attendrissement, jusqu'à me consoler de la douleur que je témoignais, et jusqu'à me demander excuse d'avoir douté (II, p. 92).

Jamais plus Jacob ne pourra retrouver sa sincérité. Conduit par sa complaisance, ici, par exemple, il feint de n'avoir point d'appétit alors qu'il est en vérité affamé (III, p. 118), là il plaque des gestes galants sur son comportement naturel (III, p. 120).

Mais la métamorphose ne s'arrête pas là. Au fur et à mesure qu'il gravit les échelons de son ascension sociale, ses tours de gibecière dénaturent toujours davantage son identité, son langage corporel devient le produit d'une étude et d'un calcul de plus en plus systématiques. Comme l'indique D. J. Adams, "the higher Jacob rises in society, the greater will be the pressure on him to develop and present a controlled, constant image if he is to succeed in his ambitions " 28 .

Le personnage qu'il est devenu n'a plus rien de commun avec ce qu'il était à l'origine. Il s'est opéré une distinction fondamentale entre l'enfant Jacob, qu'il révèle dans l'intimité, et l'homme Monsieur de la Vallée, qui se masque et se couvre lorsqu'il s'offre à la société. Ce dédoublement de la personnalité est d'ailleurs symbolisé lors d'une nouvelle phase de sa métamorphose, celle où la cadette lui donne un équipage de chevalier pour remplacer ses vêtements de domestique. L'" habit uni et sans livrée" (I, p. 15) qui était le sien à un moment où on pouvait

28 D. J. Adams, "Society and Self in Le paysan parvenu", Forum for Modern Language Studies, $\mathrm{n}^{\circ} 14,1978$, p. 383. 
supposer que Jacob possédait encore une part de son unité psychologique est substitué par des vêtements caractérisés par une "doublure de soie" (III, p. 166) qui lui fait battre le coeur: "j'eus la joie de voir Jacob métamorphosé en cavalier, avec la doublure de soie, avec le galant bord d'argent au chapeau, et l'ajustement d'une chevelure qui me descendait jusqu'à la ceinture" (III, p. 167) ${ }^{29}$.

Ainsi, au fur et à mesure de sa progression sur l'échelle sociale, Jacob/La Vallée se laisse-t-il emporter par "un tourbillon de vanité [...] flatteuse". À ses yeux perdus et égarés, à son regard qui s'illusionne sur lui-même, il imagine que seule l'"école de mollesse, de volupté [et] de corruption" qu'est le monde peut conduire au raffinement de son âme ${ }^{30}$. Répondant à la lettre aux règles de cette école, il devient "méconnaissable" 31 : "moi-même je me trouvais tout autre" (IV, p. 188).

Bien heureusement pour lui, cette distance qui progressivement s'agrandit entre son être et son vouloir-être n'empêche pas qu'il garde auprès des femmes tout son ascendant. En fait, cellesci peuvent bien lui pardonner toute la mécanique de sa métamorphose parce qu'elles savent bien que dans l'espace intime Jacob redevient tout à coup leur enfant.

Sans conséquence lorsqu'il se trouve protégé des regards d'autrui, le miroir des illusions sera en miettes fracassé au moment où il retournera sur la scène de la vie sociale. L'équilibre entre son identité individuelle et son identité sociale devient d'une précarité telle que Jacob ne peut le maintenir. L'écart entre les lieux de sa personnalité s'élargit, s'agrandit, s'ouvre devant lui tel un abîme, un gouffre: c'est un néant vers lequel il est attiré inéluctablement. Il ne peut rabattre un pont qui rejoindrait les deux rives, il ne sait ajuster son comportement non verbal. Voilà ce qui se produit lorsque le comte d'Orsan l'invite à monter dans son carrosse:

je montai en carrosse, incertain si je devais y monter le premier, et n'osant en même temps faire des compliments là-dessus. Le

29 Le rapport que nous établissons entre la "doublure de soie" et le dédoublement de la personnalité est redevable à J. A. Fleming, "Textual Autogenesis in Marivaux's Paysan parvenu", Studies on Voltaire and the Eighteenth Century, no 189, 1980, p. 198-199.

30 "[L]'âme se raffine à mesure qu'elle se gâte", croit-il (IV, p. 187).

31 "[J]e devins méconnaissable, tant j'acquis d'éducation et d'expérience" (IV, p. 187). 
80

savoir-vivre veut-il que j'aille en avant, ou bien veut-il que je recule? me disais-je en l'air, c'est-à-dire en montant. Car le cas était nouveau pour moi, et ma légère expérience ne m'apprenait rien sur cet article; sinon qu'on se fait des cérémonies lorsqu'on est deux à une porte, et je penchais à croire que ce pouvait être ici de même (V, p. 261).

Jacob ne sait plus sur quel pied danser dans la ronde des conventions. La blessure de son anéantissement apparaît de façon encore plus tragique lors de la dernière scène du roman, au cours de laquelle il est conduit par son nouvel ami dans le lieu sacré de la Comédie, symbole extrêmement riche et condensé de tout ce theatrum mundi si caractéristique de la société de l'époque. Le comte d'Orsan s'y sent "aussi à [s]on aise que dans [s]a chambre", puisque le masque est pour lui une double peau, mais c'est loin d'être le cas du pitoyable Jacob. En fait, avoue-t-il, "les airs et les façons de ce pays-là me confondirent et m'épouvantèrent": "Hélas! mon maintien annonçait un si petit compagnon, je me voyais si gauche, si dérouté au milieu de ce monde qui avait quelque chose de si aisé et de si leste! Que vas-tu faire de toi? me disais-je" (V, p. 265).

Jacob se sent complètement perdu, dérouté, décontenancé, embarrassé. Plein de remords et de culpabilité, il se met à éprouver "une confusion secrète de [s]e trouver là": "c'était [...] un certain sentiment de mon indignité qui m'empêchait d'y être hardiment, et que j'aurais bien voulu qu'on ne vît pas dans ma physionomie, et qu'on n'en voyait que mieux, parce que je m'efforçais de le cacher" (ibid.).

Mes yeux m'embarrassaient, je ne savais sur qui les arrêter; je n'osais prendre la liberté de regarder les autres, de peur qu'on ne démêlât dans mon peu d'assurance que ce n'était pas à moi à avoir l'honneur d'être avec de si honnêtes gens, et que j'étais une figure de contrebande; car je ne sache rien qui signifie mieux ce que je veux dire que cette expression qui n'est pas trop noble.

Il est vrai aussi que je n'avais pas passé par assez de degrés d'instruction et d'accroissements de fortune pour pouvoir me tenir au milieu de ce monde avec la hardiesse requise. J'y avais sauté trop vite; je venais d'être fait monsieur, encore n'avais-je pas la subalterne éducation des messieurs de ma sorte, et je tremblais qu'on ne connût à ma mine que ce monsieur-là avait été Jacob (V, p. 265-266). 
Exposé au regard d'autrui, sa "figure de contrebande" est mise au jour, dépouillée de son masque factice, il n'offre plus qu'une "hétéroclite figure", si ridicule qu'elle lui fait dire: "qu'il n'y avait rien de si sot que moi, ni de si plaisant à voir " (V, p. 266). Aux questions qu'on lui adresse, il formule des réponses déplacées et un comportement non verbal qui ne l'est pas moins. Écartelé entre son moi enfant et son moi adulte, incapable d'assumer ce dernier, le garçon n'est rien de moins qu'un fantoche grotesque et un risible pantin:

Voilà de mes réponses, que j'accompagnais civilement de courbettes de corps courtes et fréquentes, auxquelles apparemment ces messieurs prirent goût, car il n'y en eut pas un qui ne me fît des compliments pour avoir la sienne.

Un d'entre eux que je vis se retourner pour rire me mit au fait de la plaisanterie, et acheva de m'anéantir [...] (ibid.).

Certes Jacob échappera à l'insupportable lorsque, conduit au théâtre par le comte d'Orsan, il sera sauvé par la foule où il se confondra, où il deviendra anonyme. Mais Marivaux ne pourra résoudre son dilemme, ne pourra concilier les deux parties divisées de son héros. Il laisse donc son roman inachevé.

\section{L'échec comme prix de la réussite}

Dans les écrits qui ont précédé la parution du Paysan parvenu, Marivaux prônait avec véhémence l'adoption d'un comportement non verbal sincère, répondant au code du sentiment, et qui battrait en brèche une fois pour toutes les mensonges du comportement conventionnel. Ce qui importait pour lui était que l'on "se ressemble fidèlement", qu'on refuse d'entrer dans le jeu de la falsification et de l'aliénation de son individualité et qu'on participe ainsi à la mise en place de rapports humains fondés sur la vertu, l'honnêteté et la franchise.

Jacob avait d'ailleurs lui-même insisté sur l'importance de se conformer aux règles de cette morale. Il avait amorcé son récit par de virulents propos liminaires au cours desquels il attaquait tous ceux qui voient dans la naissance et le rang les seuls mérites dignes de considération. Ces hypocrites, il avait été jusqu'à les qualifier de "sots" (I, p. 5). Croyant que c'est "une erreur, au reste, que de penser qu'une obscure naissance vous avilisse", il suggérait de ne rien cacher et d'avouer franchement la nature de 
82

son état véritable. Cette stratégie, selon lui, aurait pour conséquence de mettre un terme aux sarcasmes: "la malignité des hommes vous laisse là; vous la frustrez de ses droits; elle ne voudrait que vous humilier, et vous faites sa charge; vous vous humiliez vous-même, elle ne sait plus que dire" (I, p. 6).

Toujours dans son préambule, il ne fait aucun quartier à ceux qui dérogent à cette règle de franchise. Il se montre impitoyable à l'endroit de ceux qui, pour se mettre "à couvert du dédain du monde", ont "la faiblesse de rougir eux-mêmes de leur naissance, de la cacher, et de tâcher de s'en donner une qui embrouillât la véritable" (I, p. 5-6). Des paroles. Que de vaines paroles.

Comment, lui, s'est-il comporté sinon en histrion, objet de son propre dégoût? Enivré par son succès, illusionné par ses ambitions, il n'a fait rien d'autre que de s'écarter de la sincérité qu'il proposait. Il a été incapable de faire autrement que de devenir étranger à luimême. Progressivement il est entré dans le monde des usages que Parménie redoutait tant. Progressivement il s'est laissé corrompre par la société civile. Et en bout de ligne il a eu la faiblesse de rougir de sa naissance, il a eu l'infâmie de s'en attribuer une autre.

Le roman n'a pas été terminé parce qu'il ne pouvait l'être. Jacob l'avait lui-même annoncé. Il l'avait prédit dès la deuxième page de son récit, à propos de l'artifice qui consiste à cacher sa naissance et à s'en donner une autre:

cet artifice-là ne réussit presque jamais; on a beau déguiser la vérité là-dessus, elle se venge tôt ou tard des mensonges dont on a voulu la couvrir; et l'on est toujours trahi par une infinité d'événements qu'on ne saurait ni parer, ni prévoir; jamais je ne vis, en pareille matière, de vanité qui fît une bonne fin.(I, p. 6, souligné par nous).

Connaissant mieux que quiconque l'histoire de sa vie, celle qu'il s'apprête à raconter, Jacob sait qu'arrivera un moment où elle tournera à la catastrophe. Il sait qu'il sera trahi par des événements qu'il n'a pu prévoir. En cette matière, la vanité ne peut donner de bonne fin. Elle ne le peut parce que le paysan parviendra malgré tout, et que pour parvenir dans le monde qu'il décrit, il devra se montrer toujours plus vain, toujours plus hypocrite, toujours plus menteur; il devra se transformer en monstre, devenir l'incarnation de l'imposture humaine.

Retournons à la case départ. Relisons les premières lignes du roman. Jugeons si le narrateur n'est pas un imposteur. "Le titre 
que je donne à mes Mémoires, écrit-il, annonce ma naissance; je ne l'ai jamais dissimulée à qui me l'a demandée, et il semble qu'en tout temps Dieu ait récompensé ma franchise là-dessus; car je n'ai pas remarqué qu'en aucune occasion on en ait eu moins d'égard et moins d'estime pour moi" (I, p. V). Nous savons ce qu'il en est de cette franchise. Nous savons que Jacob n'a cessé d'amplifier progressivement son mensonge, et que ce mensonge l'a couvert de ridicule. D'ailleurs, comment Jacob peut-il prétendre à la sincérité lorsqu'il refuse au lecteur fictif de lui révéler son nom? "Pour mon nom, je ne le dis point: on peut s'en passer; si je le disais, cela me gênerait dans mes récits" (I, p. 7).

Marivaux n'a pas terminé son roman. Il ne l'a pas terminé parce qu'il a réalisé que son contrat social, sa morale fondée sur la sincérité du comportement, non verbal et verbal, n'était autre chose qu'une vaste utopie, qu'elle ne pouvait être mise en pratique. Il n'a pas terminé parce que, malgré ses intentions, il a éprouvé que "toute conduite dans laquelle une tendance isolée de l'esprit humain tente de s'imposer aux autres d'une manière fixe et définitive, se change en son opposé". Hegel disait que toute vision morale du monde que l'on propose à l'autre repose sur des contradictions, et que c'est "au cours de son expérience, dans l'épreuve de l'action", qui est ici action d'écrire, que les contradictions se révèlent à la conscience. Dans un tel cas "la conscience morale devra renoncer à son moralisme ou consentir à être ce qu'elle ne croyait pas être "32. Marivaux n'était pas prêt à faire cette concession. Il préfère accepter son échec. C'est le prix qu'il avait à payer pour la réussite de Jacob.

32 Cité par Jacques d'Hondt, "Hegel et Marivaux", Europe, nos 451-452, novembre-décembre 1966, p. 334-335. 